\title{
Type 2 Diabetes Predicts Increased Risk of Neurodegenerative Complications in Veterans Suffering Traumatic Brain Injury
}

\author{
Mark B. Zimering1,2*, Deesha Patel ${ }^{1}$, Gideon Bahn ${ }^{3}$ \\ ${ }^{1}$ Endocrinology, Veterans Affairs New Jersey Healthcare System, East Orange, NJ \\ ${ }^{2}$ Rutgers-Robert Wood Johnson Medical School, New Brunswick NJ, USA
}

${ }^{3}$ Hines Veterans Affairs Hospital, Hines, Illinois

\begin{abstract}
Received: October 17,2019; Accepted:November 14,2019; Published: November 20,2019
*Corresponding author : Mark B. Zimering, Endocrinology, Veterans Affairs New Jersey Healthcare System, East Orange, NJ and Rutgers-Robert Wood Johnson Medical School, New Brunswick NJ, Medical Service (111); 151 Knoll croft Rd. Lyons, NJ 07939, USA; Phone: 9086470180 x4426; Fax: 908 604-5249; E-mail: mark.zimering@va.gov
\end{abstract}

\begin{abstract}
Aims

Obese type 2 diabetes and traumatic brain injury are associated with persistent peripheral and neuro-inflammation, respectively. We tested whether adult type 2 diabetes increased the hazard rate for neurodegeneration complications following traumatic brain injury.

Methods

Retrospective chart review of patients treated at the Veterans Affairs New Jersey Healthcare System between 2016-2019 and having a diagnosis of prior traumatic brain injury was performed in adult veterans, age 50 years or older. Cox proportional hazards regression analysis was used to identify risk factors predictive of an increased risk of neurodegeneration, i.e. worsening major depression, dementia or Parkinson's disease
\end{abstract} following traumatic brain injury.

\section{Results}

Type 2 diabetes predicted a nearly three-fold increased hazard ratio (HR $=2.95,95 \%$ CI 1.15-7.56, P $=0.02$ ) for the occurrence of worsening major depression, dementia or Parkinson's disease in eighty adults age 50 years or older who had experienced prior traumatic brain injury. After adjusting for other covariates, hypertension ( $\mathrm{HR}=4.15,95 \% \mathrm{CI} 1.21-14.29, \mathrm{P}=0.02)$ was significant and body mass index $(\mathrm{HR}=1.14,95 \% \mathrm{CI} 0.99$ 1.30; $\mathrm{P}=0.06$ ) modestly significant predictors of the risk for the time to first occurrence of the composite neurodegenerative outcome.

\section{Conclusion}

Type 2 diabetes, hypertension and higher body mass index increase the hazard for the occurrence of worsening depression, Parkinson's disease and dementia following traumatic brain injury in middle-aged and older adults.

Keywords: Traumatic brain injury; Type 2 diabetes mellitus; Hypertension; Neurodegeneration.

\section{Introduction}

According to the 2017 National Diabetes Statistics Report, about $10 \%$ of the United States population lives with diabetes, the vast majority have type 2 diabetes [1]. Adult type 2 diabetes mellitus is associated with an increased risk of Parkinson's disease [2], and late-onset Alzheimer's dementia (AD) [3]. Traumatic brain injury increases the risk of neurodegenerative disorders via poorly-defined mechanisms thought to involve (in part) persistent neuro-inflammation [4]. Since aging of the 'baby boomer' generation in the United States is expected to substantially increase the incidence of age-associated neurodegenerative disorders over the next several decades [5], we set out to test whether obese type 2 diabetes might increase the risk of neurodegenerative complications in middle-aged and older adult U.S military veterans who suffered a previous traumatic brain injury.

\section{Methods}

A Veterans Affairs New Jersey Healthcare System medical records database search of patients having an International Classification of Diseases (ICD)-9 and -10 diagnostic code for late effects of traumatic brain injury (TBI), diffuse traumatic brain injury (or closely-related diagnoses) was performed for the time interval 2016-2019. A retrospective chart review was conducted in which possible risk factors associated with accelerated 
cognitive decline, Parkinson's disease or worsening depression were extracted from the patient record. Patients were excluded if they had experienced any of the endpoints of dementia, Parkinson's disease or suicide attempt (severe depression) prior to the date of traumatic brain injury. The date of first TBI occurrence was recorded and subsequent occurrence (or nonoccurrence) of a neurodegenerative endpoint, i.e. Parkinson's disease, worsening major depression (i.e. suicide attempt), or dementia was used to calculate the time to first occurrence of an endpoint (or the endpoint-free observation period). The 'severity of TBI' followed the definitions used by the Defense and Veterans Brain Injury Center [6]: 'mild TBI'- loss of consciousness 0-30 minutes and alteration of consciousness from a moment up to 24 hours; 'moderate TBI'- loss of consciousness $>30$ minutes and less than 24 hours, post-traumatic amnesia more than 1 day to less than 7 days; 'severe TBI'- loss of consciousness more than 24 hours and post-traumatic amnesia more than 7 days.

Type 2 diabetes mellitus was defined as having a hemoglobin A1c $>6.4 \%$.

Major depressive disorder (MDD) required a clinical diagnosis by a psychiatrist and the current use of anti-depressant medications. Worsening depression was evidenced by suicide attempt in a patient having a prior diagnosis of major depressive disorder.

Parkinson's Disease- required clinical diagnosis by a neurologist/movement disorder specialist together with typical clinical symptoms having a positive response to L-dopa medications. Dementia- was based on clinical diagnosis by a psychiatrist or neurologist, diagnostic score on the St. Louis University Mental Status test [7] and/or the use of FDA-approved medications to treat dementia.

Hypertension- essential hypertension was based on clinical diagnosis in patients taking one or more classes of antihypertensive medications.

Plasma low-Density Lipoproteins (LDL)-Cholesterol, and triglyceride levels- the fasting plasma LDL-cholesterol and triglyceride levels recorded prior to or around the time of initiation of lipid-lowering medications was used as the risk variable value.

\section{Statistical Analysis}

We utilized Cox proportional hazard regression analyses on the time to the first occurrence of worsening major depression, dementia or Parkinson's disease comparing between diabetic patients with non-diabetic patients. For multivariate Cox proportional hazard model, we included other risk factors, age, body mass index (BMI), triglyceride, hypertension, and LDLcholesterol concentration. Two group paired t-test for continuous variables and chi-square or Fisher's exact test for dichotomous variables were examine the baseline risk factors between diabetic and non-diabetic patients. All significance test were two-sided 0.05 alpha level (SAS, verse 9.4 for Windows; SAS Institute, Cary, $\mathrm{NC})$.

\section{Results}

\section{Adults (>50 years old) having prior traumatic brain injury}

The medical records database search yielded 135 patients- all of whom had suffered a TBI. Owing to a pre-specified age cutoff of 50 year or older for inclusion in the study, fifty-four patients younger than 50 years old were excluded from the analysis. One additional patient was excluded because he had experienced dementia prior to TBI injury. Among the remaining eighty patients, $40(50 \%)$ had suffered a mild TBI, 3 (4\%) experienced a moderate TBI, 12(15\%) had a severe TBI and in $25(31 \%)$ patients TBI severity could not be determined.

\section{Baseline characteristics in the study patients}

The study group is shown in Table 1. The mean age was $64.1 \pm$ 8.1 years. Approximately one in six patients had type 2 diabetes, and nearly one half of the patients had essential hypertension. The mean level of plasma triglycerides in the study group was 243 $\pm 154 \mathrm{mg} / \mathrm{dL}$. The mean glycosylated hemoglobin concentration among type 2 diabetes patients was $8.2 \% \pm 2.5 \%$, and the range was $5.8-14 \%$. The known duration of diabetes ranged from 1-30 years, mean 7.9 years (not shown in Table 1).

Table 1: Baseline characteristics in the study patients

\begin{tabular}{|l|l|}
\hline & TBI Patients (N=80) \\
\hline Age (years) & $64.1 \pm 8.1$ \\
\hline BMI (kg/m2) & $28.7 \pm 4.1$ \\
\hline Diabetes (yes/no) & $16.70 \%$ \\
\hline Hypertension (yes/no) & $46 \%$ \\
\hline Baseline MDD (yes/no) & $61 \%$ \\
\hline Triglycerides (mg/dL) & $243 \pm 154$ \\
\hline
\end{tabular}

\section{Neurodegenerative outcome frequencies in patients with and without diabetes}

Total 8 events occurred in 13 patients with diabetes (61.5\%) compared to 12 events in 65 patients without diabetes $(18.5 \%)$ (Table 2).

\begin{tabular}{|l|l|l|}
\hline $\begin{array}{l}\text { Table 2: Neurodegenerative outcome frequency in TBI patients with } \\
\text { or without type 2 diabetes }\end{array}$ & Diabetes (N=13) & $\begin{array}{l}\text { No Diabetes } \\
(\mathrm{N}=65)\end{array}$ \\
\hline & $2 / 13$ & $1 / 65$ \\
\hline Parkinson's Disease & $4 / 13$ & $8 / 65$ \\
\hline Dementia & $2 / 13$ & $3 / 65$ \\
\hline $\begin{array}{l}\text { Severe Depression/ Suicide } \\
\text { Attempt }\end{array}$ & $8 / 13$ & $12 / 65$ \\
\hline Total Events & &
\end{tabular}

$\mathrm{N}=2$ patients were missing data on diabetes vs. no diabetes 
In two patients, a diagnosis of diabetes vs. no diabetes could not be ascertained by chart review. Dementia was the most common single neurodegenerative endpoint occurring in $4 / 13$ (31\%) of diabetic patients and $8 / 65(12 \%)$ of non-diabetic patients.

\section{Cox proportional hazards regression analysis}

In proportional hazards regression analysis (unadjusted model), type 2 diabetes was a significant predictor, hazard ratio $=2.95 ; 95 \%$ CI $1.15-7.56 ; \mathrm{P}=0.02$ of the time to first post-TBI occurrence of the composite neurodegenerative disease endpoint (worsening depression leading to suicide attempt, dementia, Parkinson's disease) (Table 3).

Table 3: Cox proportional hazards ratio: time-to-first post-TBI neurodegenerative event occurrence

\begin{tabular}{|l|l|l|l|}
\hline Variable & HR & $95 \%$ CI & P-value \\
\hline Diabetes & 2.95 & $1.15-7.56$ & 0.02 \\
\hline
\end{tabular}

$\mathrm{N}=78$ patients

\section{Association between diabetes and other baseline risk factors}

Type 2 diabetes was associated with a significantly higher cooccurrence of baseline

hypertension ( $77 \%$ vs. $42 \%, \mathrm{P}=0.02)$ compared to diabetes without hypertension (Table 4).

There was a borderline significant association $(\mathrm{P}=0.08)$ between diabetes and baseline increased body mass index (Table 4).

Table 4: Comparison of baseline risk factors in diabetic and nondiabetic patients

\begin{tabular}{|l|l|l|l|}
\hline Risk factor & Diabetes & No Diabetes & P-value \\
\hline Age (years) & $63.5 \pm 8.2$ & $64.5 \pm 8.2$ & 0.71 \\
\hline $\begin{array}{l}\text { Hypertension, N } \\
(\%)\end{array}$ & $10 / 13(77 \%)$ & $27 / 65(42 \%)$ & 0.02 \\
\hline $\begin{array}{l}\text { Triglycerides (mg/ } \\
\text { dL) }\end{array}$ & $355 \pm 317$ & $204 \pm 86$ & 0.12 \\
\hline $\begin{array}{l}\text { LDL-cholesterol } \\
(\mathrm{mg} / \mathrm{dL})\end{array}$ & $128 \pm 52$ & $136 \pm 41$ & 0.59 \\
\hline
\end{tabular}

\section{Neurodegenerative outcome frequencies in patients with and without hypertension}

Total 15 composite first neurodegenerative disease occurrence was experienced by 15 of 37 patients with hypertension (40.5\%) compared to first neurodegenerative disease occurrences experienced by five of 43 patients without hypertension (11.6\%) (Table 5).
Table 5: Neurodegenerative event frequency in TBI patients with or without hypertension

\begin{tabular}{|l|l|l|}
\hline & Hypertension $(\mathrm{N}=37)$ & $\begin{array}{l}\text { No Hypertension } \\
(\mathrm{N}=43)\end{array}$ \\
\hline Total Events & $15 / 37$ & $5 / 43$ \\
\hline
\end{tabular}

$\mathrm{N}=80$ patients

\section{Multi-variable regression analysis}

In Cox proportional hazards regression analysis that included six baseline risk factors (age, BMI, diabetes vs no diabetes, hypertension vs no hypertension, LDL-cholesterol, plasma triglyceride concentration, hypertension (vs. no hypertension) was a significant predictor $\mathrm{HR}=4.15$; $95 \%$ CI 1.21-14.29; $\mathrm{P}=$ 0.02 of the time to first post-TBI occurrence of the composite neurodegeneration endpoint (Table 6). Higher body mass index was nearly significantly predictive of the time to first event occurrence HR=1.14; 95\% CI 0.992-1.302; $\mathrm{P}=0.06$ (Table 6), after adjusting for other covariates. There was no significant interaction effect of either diabetes or hypertension and any other risk factor on the hazard for post-TBI occurrence of the composite neurodegeneration endpoint (not shown in Table 6).

Table 6: Multi-variable Cox proportional hazards ratio: time-to-first post-TBI neurodegenerative event occurrence

\begin{tabular}{|l|l|c|l|}
\hline Variable & HR & $95 \%$ CI & P-value \\
\hline Hypertension & 4.15 & $1.21-14.29$ & 0.02 \\
\hline BMI & 1.14 & $0.99-1.30$ & 0.06 \\
\hline Diabetes & 1.25 & $0.37-3.85$ & 0.72 \\
\hline Triglycerides & 1 & $0.996-1.01$ & 0.39 \\
\hline LDL-cholesterol & 0.998 & $0.986-1.009$ & 0.67 \\
\hline Age & 0.993 & $0.934-1.055$ & 0.81 \\
\hline
\end{tabular}

\section{Discussion}

The current data are the first to suggest that type 2 diabetes is associated with a substantially increased (three-fold) hazard rate for the occurrence of neurodegenerative complications in veterans following a traumatic brain injury. Prior published reports suggested that a single moderate-severe traumatic brain injury increases the risk of dementia and Parkinson's disease [8-10]. Post-mortem studies in brain from lifetime TBIsufferers indicated the presence of Lewy bodies characteristic of Parkinson's disease and Parkinson's disease with dementia and/ or neuritic plaques characteristic of AD [11-13] Chronic traumatic encephalopathy (following exposure to multiple mild TBI) is neuropathologically distinguishable from $\mathrm{AD}$ [14] consistent with the view that 'trauma-associated neurodegeneration' is likely pathophysiologically heterogeneous [15]. Since our study cohort included patients who had suffered from a single TBI (70/80 pts) and multiple mild TBIs (10/80 pts) diverse biologic pathways 
and underlying mechanisms may have contributed to composite neurodegenerative endpoint occurrence. Because information about TBI severity was missing in $30 \%$ of patients in our study cohort we did not test for a possible relationship between TBI severity and neurodegenerative endpoint occurrence.

Major depressive disorder was a highly comorbid condition occurring in $61 \%$ of the study cohort of middle-aged and older TBI patients. Major depressive disorder increases in older adult type 2 diabetes [16] and in our prior reports, circulating 5-hydroxytryptamine $2 \mathrm{~A}$ receptor-activating autoantibodies increased in subsets of obese adult type 2 diabetes suffering with major depression [17], Parkinson's disease and dementia [18]. The serotonin $2 \mathrm{~A}$ receptor is highly expressed in specific brain regions [19], e.g. medial prefrontal cortex and hippocampus dentate gyrus, implicated in depression pathophysiology and treatment responsivity, respectively. In our preliminary experiments, two older adult type 2 diabetic patients who had experienced a single moderate or severe TBI and subsequently developed dementia $(n=1)$ or Parkinson's disease $(n=1)$ both harbored 5-hydroxytryptamine $2 \mathrm{~A}$ receptor-activating autoantibodies in their circulation (Zimering MB, unpublished observations). In a recent report in forty-nine adults with type 2 diabetes mellitus, there was a significant association between circulating neurotoxic 5-hydroxytryptamine $2 \mathrm{~A}$ receptor IgG autoantibodies and the presence of one or more co-morbid microvascular (i.e. retinopathy or nephropathy) and/or neurodegenerative complications (major depression, dementia, Parkinson's disease) [20]. Hypertensive patients who had suffered cerebrovascular accident were among the patient subgroups whose IgG autoantibodies displayed highest binding in an enzyme linked immunosorbent assay using a 5-HT2A receptor, second extracellular loop region synthetic peptide as the target antigen [20].

Microvascular damage, increased blood brain barrier permeability, and chronic inflammation are pathophysiological mechanisms common to both obese, adult type 2 diabetes and traumatic brain injury [21]. Hypertension is highly co-morbid in obese adult type 2 diabetes and an independent established risk factor for accelerated cognitive decline experienced by middle-age and older populations [22]. In the Veterans Affair Diabetes Trial of 1791 middle-age and older veterans with type 2 diabetes, hypertension and hypertriglyceridemia were significant predictors of the risk of accelerated cognitive decline in executive function and processing speed [23]. These same two cognitive domains were also reported to be preferentially affected in TBI patients [24]. Abdominal obesity contributes to hypertriglyceridemia and promotes increases in proinflammatory cytokines [25] leading to chronic inflammation and increased blood brain barrier permeability [26]. The present data suggest that these and other factors associated with hypertension and obesity may contribute to the increased hazard of neurodegenerative disorder occurrences following TBI in adult type 2 diabetes.
Our findings suggest the current worldwide epidemic of obesity and type 2 diabetes [27] which is already expected to impact the future projected increase in neurodegenerative disorders incidence [5] may be particularly relevant to the 380,000 U.S. military veterans suffering with TBI [Defense and Veterans Brain Injury Center Statistics, 2000-2018][6]. In our multi-variable model, every 1 point increase in BMI, e.g. 32 to $33 \mathrm{~kg} / \mathrm{m} 2$, increases the risk of neurodegenerative outcome occurrence by $14 \%$. Obesity, type 2 diabetes and hypertension are generally modifiable risk factors whose mitigation might have profound consequences in terms of reducing the future social, economic and medical burdens of neurodegenerative disorder occurrences among lifetime TBI- sufferers.

A strength of the present study was the availability of detailed medical records permitting 'time-to-event' proportional hazards regression analysis of baseline risk factors associated with neurodegeneration following TBI. The retrospective study design means the findings are prone to recall bias inherent in the use of self-reporting. Our findings may only apply to TBI experienced by male veterans. More study in women who have higher incidences of both late-onset $\mathrm{AD}$ and major depressive disorder is needed to further evaluate the apparent association(s) between diabetes, hypertension and higher BMI with an increased risk of neurodegenerative disorders occurrences following TBI. Metabolic syndrome is prevalent in obese adult type 2 diabetes and it is associated with additional non-glycemic factors which may independently drive neurodegeneration including: abdominal obesity, systolic hypertension, hypertriglyceridemia and micro-albuminuria.

In summary, these are the first data suggesting a possible role for adult type 2 diabetes, hypertension and increased body mass index in the increased risk for common forms of neurodegeneration occurrence following TBI. The findings have significance to US military veterans who experience lifestyle changes, i.e. increased sedentariness, and weight gain, after deployment in part due to the high rate of psychological trauma and treatment with anti-depressant medications. More study of the mechanisms underlying the link between diabetes and accelerated cognitive decline in older veterans could lead to improved strategies to prevent late occurrence of neurodegenerative sequelae in younger veterans affected by TBI.

\section{Acknowledgments}

New Jersey Commission on Brain Injury Research, (Trenton, NJ) for grant support (MBZ).

The views expressed here are solely those of the authors. 


\section{References}

1. CDC. National Diabetes Statistics Report. 2017;

2. Hu G, Jousilahti P, Bidel S, Antikainen R, Tuomilehto J. Type 2 Diabetes and the Risk of Parkinson's Disease. Diabetes Care. 2007; 30(4):842847.

3. Cheng G, Huang C, Deng $H$, Wang $H$. Diabetes as a risk factor for dementia and mild cognitive impairment: a meta-analysis of longitudinal studies. Intern Med J. 2012;42(5):484-491. doi: 10.1111/j.1445-5994.2012.02758.x.

4. Walker KR and Tesco G. Molecular mechanisms of cognitive dysfunction following traumatic brain injury. Frontiers in Aging Neuroscience. 2013;5:29. doi:10.3389/fnagi.2013.00029.

5. Brookmeyer R, Gray S, Kawas C. Projections of Alzheimer's disease in the United States and the public health impact of delaying disease onset. Am J Public Health. 1998; 88(9):1337-1342.

6. DoD numbers for TBI worldwide, https://dvbic.dcoe.mil/system/ files/tbi-numbers/worldwide-totals-2000-2018Q1-total_jun-212018_v1.0_2018-07-26_0.pdf

7. Tariq SH, Tumosa N, Chibnall JT, Perry MH, Morley JE. Comparison of the Saint Louis University mental status examination and the Mini-Mental State Examination for detecting dementia and mild neurocognitive disorder-A pilot study. Am J Geriatr Psychiatry. 2006;14(11):900-910. doi:10.1097/01.JGP.0000221510.33817.86

8. Mendez MF. What is the Relationship of Traumatic Brain Injury to Dementia? J Alzheimers Dis. 2017;57(3):667-681. doi: 10.3233/ JAD-161002.

9. Plassman BL, Havlik RJ, Steffens DC, Helms MJ, Newman TN, Drosdick D. Documented head injury in early adulthood and risk of Alzheimer's disease and other dementias. Neurology. 2000;55(8):1158-1166.

10. Goldman SM, Tanner CM, Oakes D, Bhudhikanok GS, Gupta A, Langston JW. Head injury and Parkinson's disease risk in twins. Ann Neurol. 2006;60(1):65-72.

11. Crane PK, Gibbons LE, Dams-O'Connor K, Trittschuh E, Leverenz JB, Keene CD, et al. Association of Traumatic Brain Injury With Late-Life Neurodegenerative Conditions and Neuropathologic Findings. JAMA Neurol. 2016;73(9):1062-1069. doi: 10.1001/ jamaneurol.2016.1948

12. Kenney K, Iacono D, Edlow BL, Katz DI, Diaz-Arrastia R, Dams O'Connor K, et al Dementia After Moderate-Severe Traumatic Brain Injury: Coexistence of Multiple Proteinopathies. J Neuropathol Exp Neurol. 2018;77(1):50-63. doi: 10.1093/jnen/nlx101.

13. Johnson VE, Stewart W, Smith DH. Widespread $\tau$ and amyloid- $\beta$ pathology many years after a single traumatic brain injury in humans. Brain Pathol. 2012;22(2):142-149. doi: 10.1111/j.17503639.2011.00513.x

14. Takahata $\mathrm{K}$, Tabuchi $\mathrm{H}$, Mimura M. Late-onset Neurodegenerative Diseases Following Traumatic Brain Injury: Chronic Traumatic Encephalopathy (CTE) and Alzheimer's Disease Secondary to TBI (AD-TBI). Brain Nerve. 2016;68(7):849-857. doi: 10.11477/ mf.1416200517.
15. Washington PM, Villapol S, Burns MP. Polypathology and dementia after brain trauma: Does brain injury trigger distinct neurodegenerative diseases, or should they be classified together as traumatic encephalopathy? Exp Neurol. 2016;275 Pt 3:381-388. doi: 10.1016/j.expneurol.2015.06.015.

16. Anderson RJ, Freedland KE, Clouse RE, Lustman PJ. The prevalence of comorbid depression in adults with diabetes: a meta-analysis. Diabetes Care. 2001; 24(6):1069-1078.

17. Zimering MB. Diabetes Autoantibodies Mediate Neural- and Endothelial Cell- Inhibitory Effects Via 5-Hydroxytryptamine- 2 Receptor Coupled to Phospholipase C/Inositol Triphosphate/Ca2+ Pathway. J Endocrinol Diab. 2017; 4(4):1-10. DOI: 10.15226/2374$6890 / 4 / 4 / 00184$

18. Zimering MB. Circulating Neurotoxic 5-HT2A Receptor Agonist Autoantibodies in Adult Type 2 Diabetes with Parkinson's Disease. J Endocrinol Diabetes. 2018;5(2):1-11. doi: 10.15226/23746890/5/2/01102.

19. Xu T and Pandey SC. Cellular localization of serotonin (2A) (5HT (2A)) receptors in the rat brain. Brain Res Bull. 2000; 51(6):499-505.

20. Zimering MB. Autoantibodies in Type-2 Diabetes having Neurovascular Complications Bind to the Second Extracellular Loop of the 5-Hydroxytryptamine 2A Receptor. Endocrinol Diabetes Metab J. 2019;3(4): 118.

21. Michael W. Weiner, Karl E. Friedl, Anthony Pacifico, Julie C. Chapman, Michael S. Jaffee, Deborah M. Little, et al. Military risk factors for Alzheimer's disease Alzheimers Dement. 2013; 9(4): 445-451. doi: 10.1016/j.jalz.2013.03.005

22. Swan GE, DeCarli C, Miller BL, Reed T, Wolf PA, Jack LM, et al. Association of midlife blood pressure to late-life cognitive decline and brain morphology. Neurology. 1998; 51(4):986-993.

23. Zimering MB, Knight J, Ge L, Bahn G. VADT Investigators. Predictors of cognitive decline in older adult type 2 diabetes from the veterans affairs diabetes trial. Front Endocrinol (Lausanne). 2016; 7:123.doi: 10.3389/fendo.2016.00123

24. Kaup AR, Peltz C, Kenney K, Kramer JH, Diaz-Arrastia R, Yaffe K. Neuropsychological Profile of Lifetime Traumatic Brain Injury in Older Veterans. J Int Neuropsychol Soc. 2017;23(1):56-64.doi: 10.1017/S1355617716000849

25. Jung UJ and Choi MS. Obesity and its metabolic complications: the role of adipokines and the relationship between obesity, inflammation, insulin resistance, dyslipidemia and nonalcoholic fatty liver disease. Int J Mol Sci. 2014; 15(4):6184-6223. doi: 10.3390/ijms15046184

26. O'Carroll SJ, Kho DT, Wiltshire R, Nelson V, Rotimi O, Johnson R, et al. Pro-inflammatory $\mathrm{TNF} \alpha$ and IL-1 $\beta$ differentially regulate the inflammatory phenotype of brain microvascular endothelial cells.J Neuroinflammation. 2015;12:131. doi: 10.1186/s12974-015-03460 .

27. Wild S, Roglic G, Green A, Sicree R, King H. Global prevalence of diabetes: estimates for the year 2000 and projections for 2030. Diabetes Care. 2004; 27(5):1047-1053. 